\title{
Of Remedies and Poisons: Recreational Use of Antiretroviral Drugs in the Social Imagination of South African Carers
}

\author{
*Fiona Larkan \\ Department of Anthropology, National University of Ireland (NUI) Maynooth \\ E-mail address:fiona.larkin@nuim.ie \\ Brian Van Wyk \\ School of Public Health, University of the Western Cape, South Africa \\ E-mail address:branwyk@uwc.ac.za \\ Jamie Saris \\ Department of Anthropology, National University of Ireland (NUI) Maynooth \\ E-mail address:ajamie.saris@nuim.ie
}

\begin{abstract}
During an ethnographic study of barriers to, and compliance with, antiretroviral (ARV) treatment in the South Africa's West Coast region, our team came across a general sense amongst heath care providers that there was a lively illicit trade in antiretroviral medications. In itself, this is seen to be a barrier to adherence for many of their patients whose medication is traded to, or stolen by, drug dealers. Independent anecdotal evidence is emerging about this trade, though there has been little hard data verifying the existence of a recreational market for ARVs. While there are rumours that Efavirenz (some of whose side effects are hallucinogenic) is being used in the manufacture of crystal methamphetamine (locally 'tik'), such reports, in themselves, do not seem able to explain the ubiquity (and the confidence) of the belief in this trade amongst the health care providers with whom we have interacted. This paper explores aspects of the off-label trade of ARVs (as we have come to know it) and, as importantly, how rumor and knowledge of this trade has gained increasing currency in the social imagination of health and social care workers. This, we argue, could precipitate a real crisis in the Government's public rollout programme.
\end{abstract}

Keywords: Social Imagination, South Africa, antiretroviral treatment, Recreational drugs, HIV/AIDS

*Corresponding author - fiona.larkan@nuim.ie 


\section{Introduction}

This paper concerns the reported off-label usage of Efavirenz (brand-named Stocrin in South Africa) and other antiretrovirals (ARVs) in the Western Cape Province of South Africa. We explore some of the evidence for this off-label usage, as well as how understandings of this usage have become embedded in what we call the social imagination of Health Care Workers in the area. Finally, we explore some of the implications of such reports for the antiretroviral programme rollout.

Globally, South Africa has the largest public-funded Antiretroviral Treatment (ART) programme, serving almost one million HIV-positive patients from 500 public health facilities across the country. In many respects, this system is considered the model for the rest of the Developing world. ARVs are available free of charge to any HIV-positive adult or adolescent who meets the criteria (below) ${ }^{1}$. However, despite the availability of ARV drug treatment through South Africa's public health system since 2004, two thirds of patients in need cannot or do not access treatment (WHO, 2007). A further proportion cannot or do not adhere to treatment once accessed.

A two-year ethnographic study has been underway in South Africa's West Coast region since January 2009, which seeks to understand barriers to access and adherence to ARV medicines. This study operates from the premise that such barriers, much like the diseases being treated, are the result of the complex intertwining of political, economic, social-cultural, gender, and biological factors.

The issue of an off-label trade of ARVs was first brought to the attention of researchers by home-based care workers who, it was found, would constantly introduce the topic in the context of more general discussions about social grants, poverty, crime rates and gang activities in the area. Given the serious implications such a trade could have not only on the biological outcomes of HIV-positive patients, but on future funding, it was felt that the topic merited further research. In particular it was felt that focus group discussions could explicate the extent and operations of any market that might exist and the supply and demand factors that fed any such trade.

\section{Background}

ARVs in South Africa are available free of charge to any HIV-positive adult or adolescent with a CD4 count of less than 200 (or $<350$ in the case of pregnant women or patients with TB); OR to anyone who has reached the WHO measured Stage IV of an AIDS-defining illness, irrespective of CD4 count; OR anyone with MDR or XDR TB irrespective of CD4 count (DoH, 2010). Prior to initiation on treatment (during what is known as the 'work-up' phase) patients need to meet psychosocial criteria as certified by an appropriate counsellor whose assessment is based both on interactions 1 Different criteria apply for HIV-positive children, who were outside the scope of this study. 
with the client, and the formal and informal knowledge that they have about the client. It is the informal knowledge that counsellors gain about clients that gives opportunity for rumour, gossip and hearsay to be considered as part of the client's suitability for inclusion onto the programme. These criteria, which are specifically not intended to be exclusion criteria, are:

- Demonstrated reliability, i.e. has attended three or more scheduled visits to an HIV clinic.

- No active alcohol or other substance abuse.

- No untreated active depression.

- Disclosure: It is strongly recommended that clients have disclosed their HIV status to at least one friend or family member OR have joined a support group.

- Insight: Clients need to have accepted their HIV-positive status, and have insight into the consequences of HIV infection and the role of ARV treatment before commencing ARV therapy.

- Able to attend the antiretroviral centre on a regular basis (transport may need to be arranged for patients in rural areas or for those remote from the treatment site).

The recommended first line treatment regime for patients who meet the above criteria is also clearly defined in the national government guidelines. A central component of the first-line drug cocktail is a non-nucleoside reverse transcriptase inhibitor drug Efavirenz/Stocrin. Reported common side effects of Efavirenz/Stocrin include psychiatric symptoms such as insomnia, confusion, memory loss, and depression (Cespedes et al, 2006) and more serious symptoms such as psychosis may occur in patients with compromised liver or kidney function (Hasse et al, 2005; Louwenhaupt et al, 2007).

\section{Methodology}

The field site in which our ethnographic research project is underway includes three antiretroviral treatment (ART) clinics which serve a (largely rural) area of the Western Cape Province of some $450 \mathrm{~km}$ radius. Up to 30 Primary Health Care (PHC) clinics feed into the three ART clinics. In addition, each ART clinic works with at least two non-governmental organsiations (NGOs) which employ home-based carers to visit vulnerable patients in their homes to assess their needs and, crucially, to support them in their treatment regimen. Over a two year period thus far a team of researchers have conducted extended interviews with over 120 patients. Where patients gave their permission they were 'shadowed' by a research team member as they negotiated the treatment pathway and consulted with a range of nurses, doctors, adherence counsellors, treatment counsellors and home-based carers or community workers. In addition, many patients gave their permission to repeated interviews and visits to their homes for meetings with family members/friends or other members of 
their support network. Parallel to the patient interactions, repeated, extended semistructured interviews were conducted with over 30 health workers including clinicians, nurses, pharmacists, counsellors and community care workers, all of whom were working within the public ARV rollout schemes. All interviews were conducted in the language of choice of the participant (usually English, Afrikaans or isiXhosa, which are the three primary languages of the region), were digitally recorded, transcribed in full and translated into English which is the working language of the research team. Detailed field notes and field diaries were kept by the researchers and form part of the data.

Finally, multi-lingual focus group discussions which form the basis for this paper were held with two groups of home-based carers and community health workers - one in each of two ART sites. These discussions were conducted in English, Afrikaans and isiXhosa as participants were encouraged to speak in whichever language they were most comfortable with. Although English was the common language of all present, it is rarely a first language. Researchers who were fluent in all three languages were present to translate immediately. Again, all discussions were digitally recorded, transcribed and translated into English.

\section{Social Imagination}

While there is some evidence for 'off-label' use of ARVs, we have been struck by how certainty of such use has begun to enter into what we call the social imagination of this Western Cape community of carers over the past 18 months to two years. But what do we mean by 'social imagination'? Broadly speaking the social imagination operates in the interstices between private thought and personal experience, and systemic forces ranging from trends in thought to structural conditions. In that sense, the social imagination can be seen to occupy the middle ground between individuals, and systems and structures. Mills (1959) saw social imagination primarily as private individuals making the connection between personal problems and the influence of broader social processes on those problems.

\section{'When people [i.e. private individuals] cherish some set of values and do not feel any threat to themselves they experience well-being. When they [private individuals] cherish values but do feel them to be threatened, they experience a crisis - either as a personal trouble or as a public issue' (Mills, 1959: 11).}

We will show how this [imagined] crisis was evoked in health care workers, when their cherished values - a clear separation between 'medicine' and 'drugs', the necessity of a rigorous compliance to the health care regime, and valorisation of a therapy that potentially makes a deadly disease a liveable state - are all threatened by poor adherence among [some] patients. 
Christens, Hanlin and Speer (2007) argue that although individuals within systems are the bearers of a social imagination, they are not entirely individually responsible for the views they hold, or for changing them. In common with them, we focus on the social imagination of the community "with an emphasis on the role of cultural beliefs, ideology and shared understandings in shaping the views that individuals hold about the systems and societies of which they are a part' (p.230). In communities such as the community of carers we are discussing here, people 'imagine' their social surroundings in an elusive set of self-understandings, stories, images, and even rumours. These stories, images, rumours and ideas, are constantly reiterated, becoming one of the ways the community makes sense of the social order, thus in Taylor's words, "paving the way for common practices and a widely shared sense of legitimacy" (2004: 23). Such social imaginations create, sustain and re-define social order within an environment of serious inequalities, persistent resource scarcities, and large social gulfs between those rendering and those in receipt of services.

The recoding of ARVs away from 'wonder drug' and towards a 'dangerous substance that is being abused' seems to be of great concern. The close association that such diverse scholars as Derrida (1970) through Martin (2006) have observed in the Pharmacon between 'remedy' and 'poison' seems to be especially worrisome. The communities in receipt of ARVs often are struggling with a history of substance abuse amongst other legacies of generations of structural violence, which is seen by many staff members to be a significant barrier not just to treatment but to better life chances. Indeed, some clinic staff are the human filter that separates those who have substance abuse issues away from those who do not as part of 'working up' a patient for ARVs according to the protocol produced above. As some staff members verge on the puritanical in their desire to isolate an ARV patient from any alcohol or drug use, not just in their own life but also in their residence, it is not surprising that they imbue the misuse of ARVs in such strong moral terms.

\section{Health Systems - the caring community:}

With the advent of antiretroviral treatment for HIV and AIDS patients, the disease profiles and illness experiences for these patients changed - mostly for the better, though many psychosocial and economic challenges remain. A HIV-positive status changed from a sure death sentence to a chronic illness (or in best circumstances, condition). However, the delayed introduction of ART in public health care in South Africa and the effervescence of HIV-related stigma and discrimination, among other things, conspired to create denialism about HIV status in many communities - which in turn manifested in a refusal to test voluntarily for HIV. Many patients, with the exception of pregnant women and children, present at public health facilities only when they become symptomatic. In these cases, the widely reported benefits of HAART - 
which is provided in SA - are curtailed. This renders HIV patients very dependent on a range of caregivers from primary health care workers to community-based carers, such as home-based carers who provide palliative care at home, and community health workers who improve the reach of the $\mathrm{PHC}$ clinic to provide adherence counselling and support to HIV/AIDS patients.

The health care system in South Africa is driven by the Alma Ata principles of the Primary Health Care Approach. The essence of this approach is that health care should give priority to the community's primary needs for health care and be responsive to community needs. In reality, however, very little outreach to the community served by the clinics is done by PHC staff themselves. Instead, this task is delegated to community health workers (CHWs). CHWs are lay people, usually from the particular community, who are trained to perform specific health related functions, without being professionally qualified to perform health care, and being compensated by what is regarded as an honorarium or stipend rather than a salary (Lewin et al, 2010). The creation of the cadre of CHWs was initially motivated by PHC staff being overloaded (Lehmann \& Sanders, 2007). Mercurially though, it is widely reported in South Africa and Africa-wide that health workers tend to refuse to acknowledge the value of CHWs to the PHC system, and rather perceive them as doing the 'dirty work' in the community that they do not have time to do. The net result is tenuous, sometimes tense, relationship between health workers (professionals) and CHWs (lay people), where PHC staff are often distant from CHWs, while at the same time being very reliant on them to receive their 'intel' about the 'community', right down to intimate details of patient's domestic situation.

The system of home-based carers, on the other hand, was instituted in response to psycho-social needs of patients in the community. They support patients who are bedridden in their homes. They are not historically linked to the health care system, but employed by the Social Welfare department. The HIV epidemic in South Africa rescaped, and brought into sharp relief, the notion of 'care' in communities, and it was soon clear that the delineation of $\mathrm{HBC}$ and $\mathrm{CHW}$ creates inefficiencies and their merger could render a more efficient service and perhaps even some cost-savings. HBCs through their supportive relationship with palliative patients in the community were seen most suited to provide adherence support to HIV and AIDS patients, but not only those who are bed-ridden. Current attempts are in place in SA to create one cadre called community-caregivers.

\section{Stories of ARV off-label trade:}

There have been several media reports of the use of Efavirenz/Stocrin as well as other ARVs for recreational purposes - whether to crush and smoke, (BBC Nerws 8.12.2008) to add during the manufacturing process of crystal methamphetamine (' $t i k$ ') (Cape Argus 20.05.2008), or to crush and add to dagga (cannabis) for smoking (locally known as 
'whoonga') (Mercury, 21.6.2010).

The recreational use of ARV drugs was explored in focus group discussions with home based carers and community health workers. They related to us the plight of patients who are desperately dependent on the welfare grants they receive from government. These temporary disability grants are given to patients for a period of six months when they are declared unfit to work on the basis of their HIV status. After six months the status of the patient grant may be reviewed.

When patients are initially put on the ART they most often present with a WHO Stage III HIV illness, i.e. they have a CD4 count of less than 200. Patients are informed that they qualify for a grant as they are medically unfit to work. The CD4 count in itself does not render a patient medically fit/unfit to qualify for a grant. However, those with low CD4 counts in most cases experience a decline in their health - hence the reporting to the health setting. It is this concomitant factor that leads to the physician motivating for the disability grant on the basis of HIV defining illness. As the importance of the CD4 count is impressed on the patient in a variety of settings they come to believe, with some justification, that a CD4 count of 200 is a sort of magic marker that determines their eligibility for a welfare grant. Patient CD4 count is checked every three months. It is envisaged that this check would indicate whether current treatment is working; soon after $A R V$ s are instituted CD4 counts can be expected to rise and viral load quashed down so low as to be undetectable. An increase in CD4 count would indicate that the person's immune system is getting stronger and HIV viral replication is suppressed. A stronger immune system would also indicate that the person is getting stronger (and potentially on the way to becoming economically productive).

Thus, the purpose of the disability grant is to aid HIV patients in recovery to become fit for gainful employment, but it is no secret that most HIV and AIDS patients in South Africa are battling unemployment and poverty. National unemployment, for example, stands at $40 \%^{2}$, but in settings where we work it can range as high as $80 \%$. For many HIV patients, therefore, the disability grants have in fact become a de facto poverty alleviation grant, used to sustain households rather than for treatment support, such as nutrition and transport to clinics. Re-application for the grant often coincides with the $2^{\text {nd }} \mathrm{CD} 4$ check and it is at this point that the patients reportedly begin to go off their ART schedule, in order to maintain their CD4 count below 200, believing this will extend the disability grant for another six months.

Our team entered a clinical context, then, where 'adherent to medication' was a complex idea, and one that could change over time. Incidences of ARVs leaking off the list were one genre of stories told about difficult patients. Thus the incidents we detail below about Efavirenz/ Stocrin being sold and used as recreational drugs, need to be understood within a broader context of anxieties about the use of ARVs by both staff

2 Figure based on proportion of employed to working age population. www.statssa.gov.za/keyindicators/QLFS (accessed 11.9.2010) 
and patients. In addition to such stories, for example, ARV drugs are also known to be sold to patients who are ill but do not want to attend their clinic, or to those who simply don't trust clinic staff with maintaining confidentiality regarding their HIV status, and to patients who are illegal immigrants - HIV-positive but not eligible for free ARVs. In other words, off-label, but therapeutic usage of medication was also reported.

Two incidences of ARV leakage that we collected directly connected such misuse to criminality. The first report is of gangs standing outside the clinics, and robbing patients of their ARV drugs, which are then sold on the black market. This scenario reportedly took place in a township near the city:

At [another suburb] where they have an ARV clinic and then the gangs wait outside. When you go inside and then they know [that the] ARV clinic HIV patients are going in there. They'll rob you from your tablets when you come out.

A second reported incident related is of a former community health worker who was mugged and robbed of medication by gang members. The victim's colleagues were convinced that this man was targeted because he was known to be carrying medicines and that the muggers knew what they were looking for. Notably in this case, there is no specific mention of $A R V s$ and the health worker was in fact carrying a wide range of medications. The connection between theft of medications and a recreational use of ARVs (rather than other types of medication) is one made by the carers themselves.

... they take actually the whole bag because there's a... they know there's some drugs. Something in that they can use for drugs... And at that time the police was driving in the street so it actually happened at that moment and he caught the guy with the... medication. That was a whole bag full of medication because we must only take the medication. We must only take the people's medication home because some of the patients can't walk. But now what happened now, he had it at the clinic. They must come fetch their medication there.

In a third account, community health workers explained that patients would voluntarily sell a range of medications (including ARVs, asthma pumps and TB medication) either directly to non-registered patients, or to drug dealers, who in turn sell it on for therapeutic or recreational use.

...not only ARV... any medication because there's all ... they sell it to the drug dealers. I heard it this morning when I was looking for that aunty I think it was in your street... [gestures to a colleague] and one of the shop's owners told me themselves they working so damn hard and this patient when they get their medication they take it to the drug dealers...they are waiting for them there. Some of them are waiting for them there even for... from the TB medication also that's what happens.

Yes even their...their asthma... their pumps they sell it for ten or thirty rand... sometimes also to people... to people who don't want to go to the clinic. I know 
about a patient of mine who's done this... I got a refusal from her husband and she isn't till today not my patient but they're staying in the same house so I would... I'm doing her blood pressure also but I know she's... selling it to a patient who also got asthma.

Yes... they use it as a drug because... there are two houses close to me... where they use and sell drugs... and there they get the ARVs from the patients... and they sell it to the drug [dealers]... who sell the drugs... and then he pays that person and sells it again in pieces to others... And then young people also use it... they walk pass that house.

One of the doctors working in an ARV clinic reported a break-in at her office where ARV drugs were the only medications stolen. This was interpreted as evidence of a recreational market for Efavirenz/Stocrin. Since then regulations to prohibit the storage of sample medication in examination rooms have been introduced at the hospital.

Finally, Gerald (51) has reported being mugged and having his bag (which contained his medication) stolen when crossing an area of open ground en route home from the clinic. However, he emphasises that it is not only his medication that is stolen, but his mobile phone, his bag and anything of value on his person. The point he makes is clear - if someone mugs him, should he automatically assume it is for his medication which is carried in an anonymous plastic bag, or is he merely the unfortunate random victim of attack.

They are stealing anything that you have on you... the medication is just in the bag when they steal it.

While none of these accounts involved direct observation of recreational use, these stories share some similarities. They clearly do not distinguish the ARVs according to their purported psychopharmacological effects - Efavirenz/Stocrin, for example is not being directly targeted. It is not clear, moreover, the precise black-market circuits these robberies are feeding: off-label therapeutic consumptions as detailed above, different therapeutic regimes (traditional/indigenous or faith-based) or recreational pleasure. Moreover, can muggings which result in the theft of medication always be interpreted as targeted attacks on the part of criminal gangs looking for ARVs for recreational use?

Only one participant reported directly experiencing the actual use of ARVs as recreational drugs. Mary (28) reports that her boy friend used to take her pills to (crush and) smoke them.

So they gave him the... those white pills and the small brown ones, but he doesn't take them... He only went once and never again... There was one time he smoked my pills. My pills!... See, it's a little pill... a little white one [indicates to Efavirenz/Stocrin on wall chart]. He smokes it ...

But now I don't know why he smokes my pills. He can, he works, he can take 
money and buy drugs and smoke them. Because like I explained to him 'Darlene said to me they're very dangerous pills. So you mustn't drink my pills, and I mustn't drink your pills. Must just leave my pills alone.' No, then he showed me he knows better, he is going to smoke it now. Now I hide my pills at my mother 'n them.

\section{Discussion}

Our findings indicate two potentially serious issues in the public experience with respect to the massification of the ART programme. The first, a historically-rooted crisis of confidence in the public health system is illustrated by people (non-registered patients) who are aware that they are living with HIV but who would rather buy their medication from other patients than register as an ART patient in the public programme. The second, a crisis of resources in the lives of those in the public programme is reflected in stories of patients who risk their own health to obtain a welfare grant (supporting therapy ostensibly restoring their economic productivity) in order to support their households that are existing at the edge of scarcity.

Stories about ARVs leaking off the list are embedded in, and in some ways help the teller work their way through, this difficult context. The ways that this complex reality is refracted through stories about the recreational use of ARVs which stress selfish pleasure-seeking and inexplicable criminality, however, are worrisome for several reasons. First is the rhetorically powerful movement of ARVs from the realm of 'gifted wonder remedy' to 'selfishly consumed poison'. Antiretroviral drugs, when used as recreational drugs (rather than medicine) are just not 'matter out of place' but can become an index of the fecklessness of a whole community. The Gift is both spurned and misused by the Receiver, and such use has the potential to produce HIV strains that are resistant to Efavirenz (amongst other elements of HAART) within this community, which would render any future ART programme with limited and more expensive options. Secondly, the ART patients who are selling their medication, either to drug dealers or to other non-registered patients, are breaking compliance with the health care regimen. Partial adherence, in addition to bedevilling their own chances of recovery, also complicates their treatment regimen, thus requiring additional health care expertise at great expense and cost to the health services. This renders the ART patient as a person 'out of place', because he fits neither within the capabilities or expertise of the ART clinic, nor within the social order created by the health care system.

Here is where the nature of the global environment in which the South African health crisis and the South African response is embedded, intersects in potentially dangerous ways with local clinical understanding of non-compliance. The psychosocial criteria that patients have to meet before initiation on ART already provide the health care workers with the potential to limit access to ARVs to those 
patients that are suspected would not be compliant to the treatment regimen. The emergence of recreational use of $A R V s$ in this social imagination has the potential to legitimise actions that are aligned with delaying access to treatment to yet more individuals who are clinically eligible. These delays make for, at best, sicker individuals beginning ARVs and, at worst, a death that was potentially avoidable. At the same time, Donor Organisations have yet another example of the intractability of giving aid to the feckless as it will only be abused or go astray leading to serious unintended consequences (a more serious HIV epidemic).

Off-label use of ARVs clearly is happening, but it is not occurring (even in the limited time that we have had a chance to investigate it) in a fashion that is amenable to explanation by logics invoking brain-chemistry or individual criminality. Further detailed ethnographic work would need to be conducted amongst the drug-using populations of the region to establish the existence and extent of recreational (ARV) drug use.

Instead these stories are embedded within the same context that structures the epidemic in the first place - a history of savage inequalities, of structured distrust of a medical system that did not always serve it poorest members well, and of individuals and communities forced to deal with scarcity as a result of their history of being at the sharp end of structural violence.

\section{Bibliography}

Cespedes MS, Aberg JA., 2006 Neuropsychiatric complications of antiretroviral therapy. Drug Safety. 29(10):865-74.

Christens BD, Hanlin CE, Speer PW., 2007. Getting the social organism thinking: strategy for systems change. American Journal of Community Psychology. 39:229-238

Derrida,J., 1981. [1970] Plato's Pharmacy. In Dissemination. [Trans Barbara Johnson].Chicago: Chicago University Press.

Department of Health., 2010. South African Antiretroviral Treatment Guidelines. Department of Health, Republic of South Africa

Hasse B, Günthard HF, Bleiber G, Krause M., 2005.Efavirenz intoxication due to slow hepatic metabolism. Clinical Infectious Diseases. 2005 Feb 1:40(3):e22-3.

Lewin SA, Babigumira SM, Glenton C, Daniels K, Bosch-Capblanch X, van Wyk BE, Odgaard-Jensen J, Johansen M, Aja G, Zwarenstein M \& Scheel I., 2010. Lay health workers in primary and community health care for maternal and child health and the management of infectious diseases. Cochrane Database of Systematic Reviews, Issue 3. Art. No.: CD004015. DOI:10.1002/14651858.CD003541.pub3. 
Lehmann U, Sanders D., 2007. Community health workers: What do we know about them? The state of the evidence on programmes, activities, costs and impact on health outcomes of using community health workers. Geneva: Evidence and Information for Policy, Department of Human Resources for Health, World Health Organization

Lowenhaupt EA, Matson K, Qureishi B, Saitoh A, Pugatch D., 2007. Psychosis in a 12-year-old HIV-positive girl with an increased serum concentration of efavirenz. Clinical Infectious Diseases. Nov 15:45(10):e128-30.

Martin, E. 2006., The pharmaceutical person. Biosocieties 1(3) 273-287

Marwaha, A. 2008., Getting High on HIV Drugs in S Africa. BBC News http://news.bbc. co.uk/2/hi/7768059.stm (last accessed September 13th 2010

Mdluli, A., Ward. B., \& Whitehorn, D., 2010 Whoonga drug spreads rapidly. In The Mercury, June $21^{\text {st }} 2010$

Mills, C. W., 1959. The sociological imagination. New York: Oxford University Press.

Prince, N., 2008 Children Smoking ARVs. In Cape Argus, May $20^{\text {th }} 2010$

Taylor, C., 2004. Modern Social Imaginaries. Durham \& London: Duke University Press.

www.statssa.gov.za/keyindicators/QLFS (accessed 11.9.201) 